\title{
Can gravitation anisotropy be detected by pendulum experiments?
}

\author{
René Verreault \\ Department of Fundamental Sciences, University of Quebec at Chicoutimi, 555 Boul. de l'Université, \\ Saguenay, $Q C$, Canada G7H 2BI \\ E-mail:Rene_Verreault@uqac.ca
}

\begin{abstract}
After some 170 years of Foucault pendulum experiments, the linear theory fails to quantitatively explain the results of any honest meticulous experiment. The pendulum motion usually degenerates into elliptical orbits after a few minutes. Moreover, unexplained discrepancies up to $\pm 20 \%$ in precession velocity are not uncommon. They are mostly regarded as a consequence of the elliptic motion of the bob associated with suspension anisotropy or as a lack of care in starting the pendulum motion. Over some 130 years, an impressive amount of talented physicists, engineers and mathematicians have contributed to a better partial understanding of the pendulum behaviour. In this work, the concept of biresonance is introduced to represent the motion of the spherical pendulum. It is shown that biresonance can be represented graphically by the isomorphism of the Poincaré sphere. This new representation of the pendulum motion greatly clarifies its natural response to various anisotropic situations, including Airy precession. Anomalous observations in pendulum experiments by Allais are analyzed. These findings suggest that a pendulum placed within a mass distribution such as the earth, the moon and the sun should be treated as an interior problem, which can better be addressed by Santilli's new theory of gravitation than by those of Newton and Einstein.
\end{abstract}

Keywords: Pendulum, gravitation, anisotropy, Poincaré sphere, biresonance, linear biresonance, circular biresonance, syzygy

\section{Introduction}

After Galileo's sentencing in 1633 for teaching, according to Copernicus's view, that the earth was rotating under a sky with fixed stars, it took 218 years for someone to come up with the first experimental proof of the planet's rotation. Jean Bernard Léon Foucault [1] had indeed noticed in 1851, while working on a lathe in his cellar, that the free end of a slender rod vibrating in a vertical plane retained its vibration direction unchanged even when the chuck was rotated by hand. Transposing that rod to a long vertical wire clamped in a fixation rigidly linked with the earth, he figured out that, from inertia, a swinging mass at the end of the vertical wire should behave similarly with an eventually rotating earth.

As early as May 1851, Airy [2], Astronomer Royal, showed that, when the pendulum bob had elliptical trajectories, the nonlinear restoring gravitational force resulted in a tendency for the ellipse to undergo precessing motion. A major improvement occurred in 1879 with the doctor thesis of a certain Heike Kamerlingh Onnes [3], who incidentally ended up, a few decades later, with the discovery of superconductivity and was awarded a Nobel Prize. Throughout his 290 pages of equations, Kamerlingh Onnes demystified some of the unwanted elliptical orbits of the pendulum as being caused by suspension anisotropy. Even today, a perfect isotropic suspension has not yet been achieved, though the principal consequences can be accounted for by calculation. Other significant contributions to the understanding of the Foucault pendulum have been made by Chessin [4], MacMillan [5], Longden [6], Noble [7], Somerville [8], Olsson [9], Latham [10], Hecht [11], Nelson et al. [12] and Pippard [13]. 
Nevertheless, all those Foucault pendulums built since 1851 were meant neither to make precise measurements of the earth rotation velocity, nor to ride between the earth and the moon [14]. Their most frequent use was to serve as an utterly simple pedagogical instrument for illustrating some basic laws of physics or, in a similar way, to artistically decorate museums and institutes while promoting science for the general public. In those implementations, the major drawback of the free (ordinary) pendulum was the rapid decay of its oscillations. In an article by Moppert et al. [15], it is stated, even some 130 years after Foucault's original performance, that "with an ordinary pendulum, a run takes at the most, three hours..." It is then not surprising that physicists and engineers took the challenge of designing systems that would sustain the oscillations indefinitely.

Pioneering work in that sense was achieved by Fernand Charron [16], professor of physics at the Université catholique d'Angers, France. The pendulum swing was amplified by synchronized magnetic impulsions (parametric amplification) until the wire would touch the inner part a friction ring (the Charron ring) placed a few centimetres below the suspension point. Any lateral movement of the wire at the end of the swing was therefore efficiently damped, so that the tendency to generate ellipses was hindered. Practically all sustained pendulums used for public demonstration incorporate such damping rings, in order to retain nice rectilinear, large amplitude oscillations. But, all in all, it seems that the free pendulum still possesses one good quality that is inherent to many untamed, unstable systems, namely some kind of hypersensitivity.

Speaking of untamed pendula, a word must be said about suspension systems. Every «textbook» pendulum is in fact a mathematical idealization where a point mass turns about a fixed suspension «point». Such a mathematical pendulum consisting of a point mass moving on a spherical surface has two degrees of freedom. However real life pendula involve a spatially extended mass moving about a mobile instantaneous center of rotation located somewhere within the strained region of a clamped wire or inside the deformed contact area of a sharp (initially) point or knife edge. The heavily strained suspension region is subject to permanent deformations which alter an essential characteristic of a pendulum: its putative constant length. Moreover, the extended bob can spin about its wire (or rod) axis, which adds a third degree of freedom with an elastic restoring torque (clamped wire) or a dissipative friction torque (point contact).

In a tentative to minimize the above problems, the French engineer Maurice Allais designed the paraconical pendulum [17], which consists of a disk (the bob) connected by a rod to a vertical stirrup whose upper inner surface holds a steel ball rolling on an optically flat steel surface. The suspension losses are significantly reduced. With that system, he made the unexpected discovery in 1954 of the so-called Allais effect. Allais used to design 30-day long experiments, day and night, restarting the pendulum every 20 minutes for 14-minute runs while allocating 6 minutes for the restart phase in the azimuth achieved at the end of the preceding run. In this way, he could detect the lunisolar periodicities of the tides in the time series of the azimuth changes at the end of the 14-minute runs. This phenomenon has not yet been explained up to now. Moreover, during one of those 30-day experiments in 1954, the pendulum showed a factor 5 change in precession speed as a total solar eclipse was passing some $1300 \mathrm{~km}$ away near Oslo [18]. Most scientists refer today to this "eclipse effect" as the Allais effect. It should be pointed out, though, that the eclipse effect and the above mentioned sensitivity to lunisolar tide periodicities should probably be considered as two distinct phenomena.

As Allais could repeat the observation of his eclipse effect in 1959, he started questioning the laws of gravitation according to Newton and Einstein. He was from then on denied credibility by the scientific establishment and he lost his financial support. That new Galileo recycled himself as an economist whose new theories earned him a Nobel Prize in 1988. After his death in 2010, a few dozen disciples $[19,20]$ 


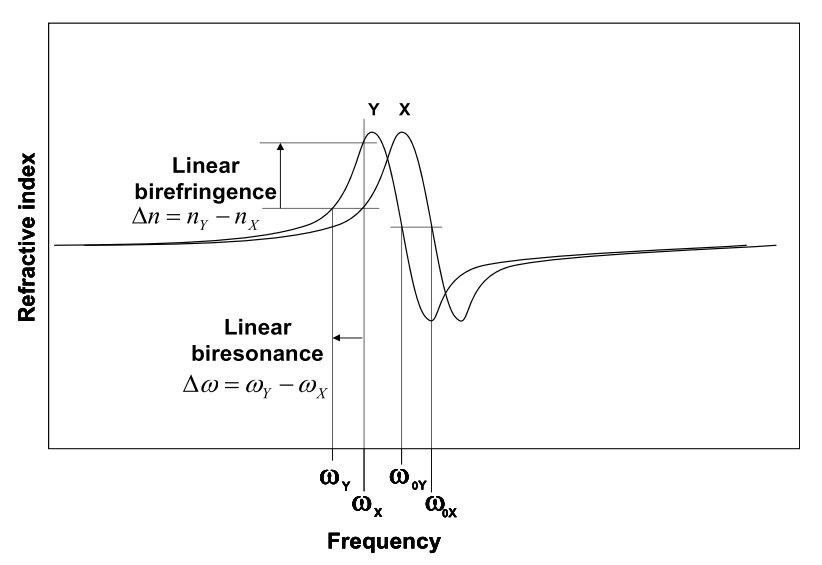

(a)

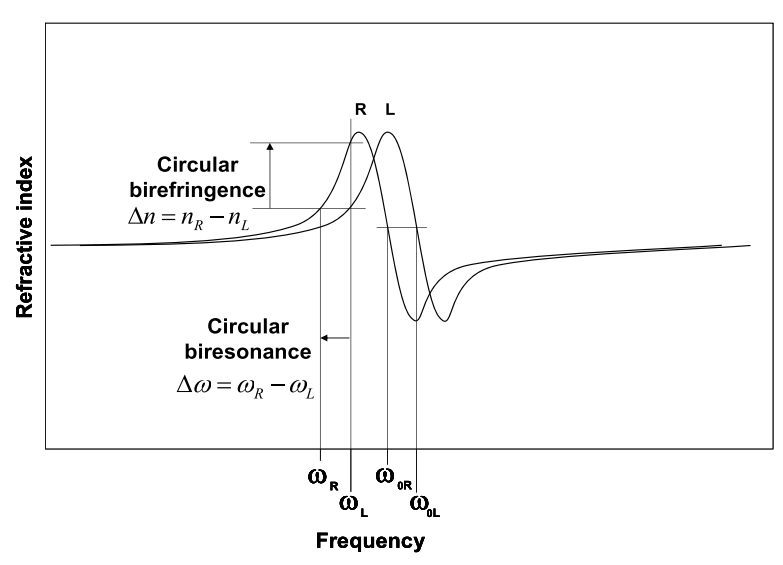

(b)

Fig. 1. Definition of linear (a) and circular (b) biresonance as a difference in resonant frequencies at constant refractive index instead of a difference in refractive indices at constant frequency for birefringence.

from the physics, mathematics and engineering communities are still chasing eclipse effects not only with paraconical and Foucault pendula, but with torsion balances [21,22] and microgravimeters [23,24].

In this paper, a new approach in describing the pendulum dynamics is presented. This sheds a new light on Allais's experiments, clarifying the complex interaction between the Foucault effect and the anisotropic properties of the pendulum environment. This is especially useful for interpreting such nonlinear pendulum response as the Airy effect and its role in Allais's findings. A new interpretation of some of Allais's results follows.

\section{Theory}

\subsection{Linear theory for harmonic oscillators}

The theory is based on an isomorphism between the oscillation states of the pendulum and the points on the surface of a sphere. It turns out that this isomorphism is a dual theory of Poincare's mathematical theory of light, published in 1892 [25]. Poincaré studied the spatial changes of the vibrating electric field when light progresses through an anisotropic medium. An illustration of the use of the Poincare sphere for describing the complex response of anisotropic crystals to magneto-optic excitation (induced circular birefringence) can be consulted in the author's dissertation [26]. Here we study the temporal changes of the mechanical vibrations of a pendulum submitted to an anisotropic potential well [27].

If one considers the dispersion curves of the refractive index about a resonant frequency of the atoms in an anisotropic crystal, there are two distinct absorption frequencies for light with two orthogonal rectilinear vibrating directions of the electric field. Classical electromagnetism considers then different resonant frequencies for the electron-nucleus system in matter, or for the system made of the electron gas versus the crystal lattice. Linear birefringence is the difference in refractive indices or the difference in velocity through matter for two orthogonal linearly polarized light vibrations $\mathrm{X}$ and $\mathrm{Y}$ at the same frequency (Fig. 1). Similarly, circular birefringence is the difference in refractive indices for two orthogonal circularly polarized light vibrations $\mathrm{R}$ and $\mathrm{L}$ at the same frequency.

Birefringence considers the polarization changes spatially along the path through the medium. Let us define linear biresonance as the difference in absorbing frequencies for two orthogonal linearly polarized 


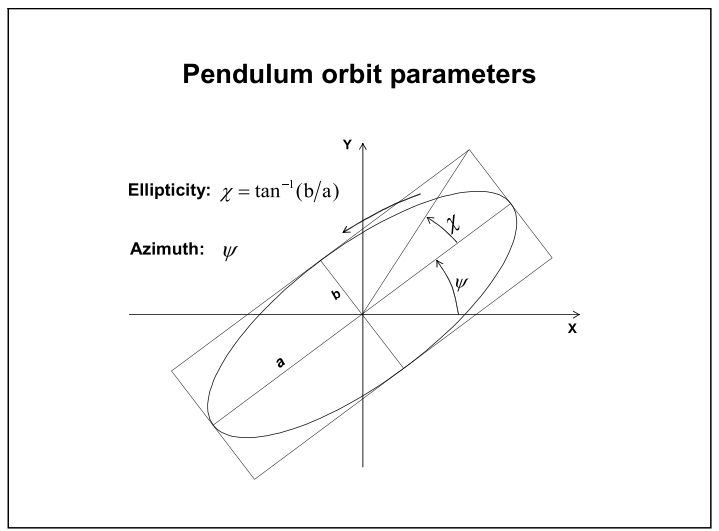

Fig. 2. Parameters of a general pendulum elliptical orbit as seen from the suspension point.

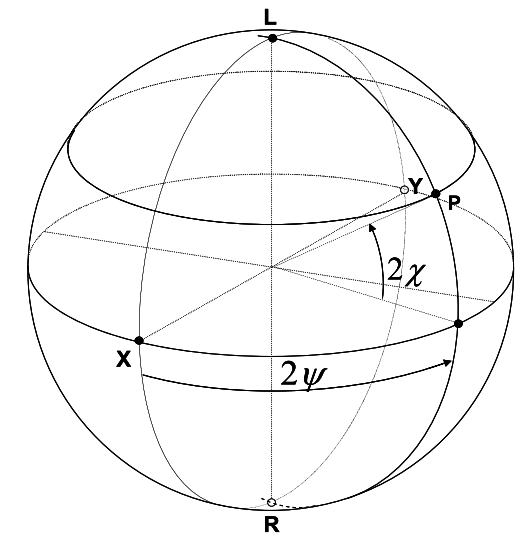

Fig. 3. The Poincaré sphere where $\mathrm{P}$ is the representative point of an elliptical orbit with azimuth $\psi$ and ellipticity angle $\chi$.

light vibrations progressing at the same speed through matter (hence with the same refractive index). Let us define circular biresonance as the difference in absorbing frequencies for two right-handed and lefthanded circularly polarized light vibrations progressing at the same speed through matter (hence with the same refractive index). Contrary to birefringence which is an environmental phenomenon at the wavelength scale, biresonance is a local phenomenon at the particle scale. It considers the local motion of the electron cloud centre of mass relative to a nucleus. That local motion ought to be rectilinear, circular or in general elliptical.

The mechanical electron-nucleus system reacts as a harmonic oscillator. The idealized point mass Foucault pendulum is also a 2-D harmonic oscillator. Hence the general oscillation state of the pendulum can be represented by the same ellipses as does polarized light, as shown by Fig. 2 .

With reference to a rectilinear vibration along the $X$-axis, the ellipse is characterized by the azimuth $\psi$ of the major axis and the ellipticity angle $\chi=\tan ^{-1}(b / a)$, the minor to major axis ratio. Finally, the direction of travel along the ellipse can be counter-clockwise (left-handed orbit) or clockwise (righthanded orbit), as seen from the pendulum suspension point.

The isomorphism between the pendulum states and the geographic coordinates on the Poincare sphere is shown in Fig. 3. The equator represents the rectilinear vibrations in such a way that the longitude is twice the oscillation azimuth $\psi$. Hence the orthogonal rectilinear states $X$ and $Y$ are diametrically opposed on the equator. The longitude of the point $\mathrm{P}$ representing an ellipse is twice the azimuth $\psi$ of the ellipse major axis. The latitude of the point $P$ is twice the ellipticity angle $\chi$. The northern hemisphere with positive ellipticities represents the left-handed elliptical pendulum orbits. The pole $\mathrm{L}$ is for the lefthanded circular orbit. The southern hemisphere with negative ellipticities represents the right-handed elliptical pendulum orbits. The pole $\mathrm{R}$ is for the right-handed circular orbit.

Figure 4 shows the evolution of a pendulum with linear anisotropy. The orthogonal rectilinear eigenstates $X$ and $Y$ which remain unchanged with time determine an eigenaxis $X Y$ in the equatorial plane. The $X$ state is taken as the fast axis (high frequency or long period of oscillation). A starting rectilinear state $P_{0}$ with azimuth at $45 \mathrm{deg}$ from the $X$ state will rotate at constant angular velocity about the $X Y$ axis as a function of time $t$. The counter-clockwise angle delta about the high frequency state $X$ is equal to the phase shift after time $t$. One sees at the pole that after a 90 degree phase shift, the orbit was a left-handed circle.

Showing the case of circular biresonance as for the Foucault effect, Fig. 5 pertains to a pendulum in the northern hemisphere of the earth. The orthogonal eigenstates of the pendulum which remain unchanged 


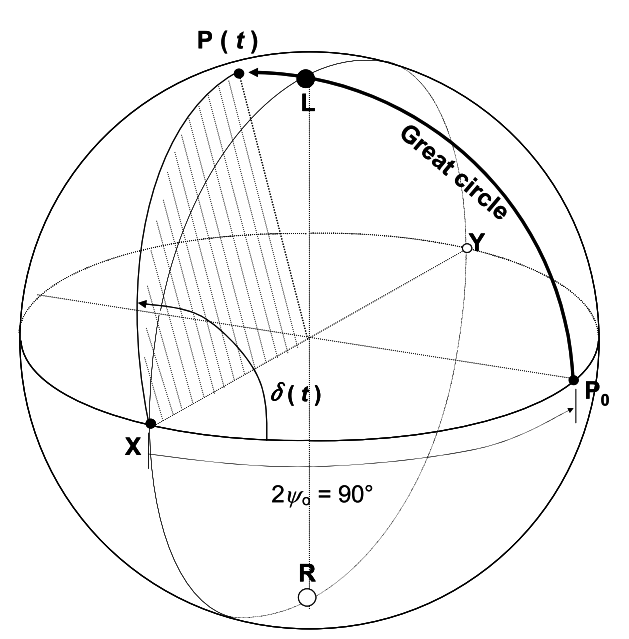

Fig. 4. Evolution of the pendulum orbit represented by point $\mathrm{P}$, with a start azimuth half way between the eigenaxes $\left(45^{\circ}\right)$, when pure linear biresonance is present. The phase angle $\delta$ grows linearly with time.

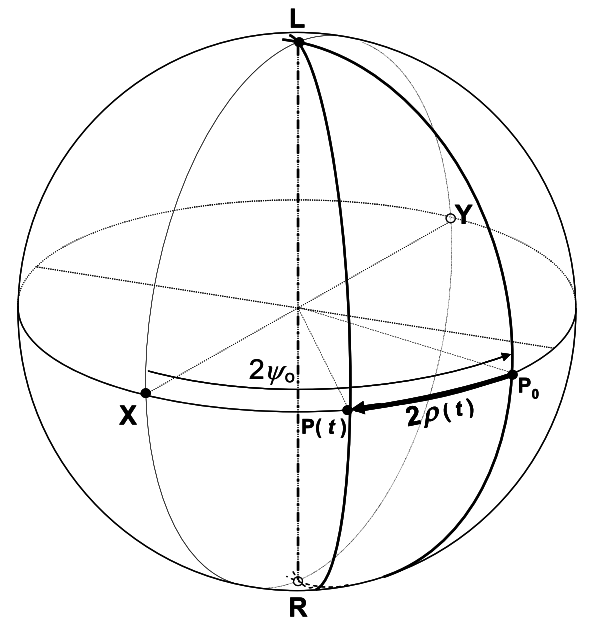

Fig. 5. Evolution of the pendulum orbit represented by point $P$, with a rectilinear starting state $\mathrm{P}_{0}$, when pure circular biresonance is present. The phase angle is twice the precession angle: $\delta(t)=2 \rho(t)$. The rotation of $\mathrm{P}$ is counter-clockwise about the faster eigenstate R, which corresponds to the Foucault effect in the northern hemisphere.

despite circular anisotropy are the two circular orbits of opposite sense. For an inertial observer airborne above the pendulum, the laboratory floor rotates counter-clockwise as well as the earth surface. Although the pendulum eigenstates are degenerate in the inertial reference frame, they have different angular velocities in the laboratory frame where the measurements of a Foucault experiment are made. As a matter of fact, due to the earth rotation, the right-handed circular orbit moves faster than the left-handed one in the laboratory frame. This causes a right-handed precession relative to the floor. On the Poincaré sphere, this is obtained through a rotation of the representative point $\mathrm{P}$ about the $R L$ polar axis by a phase angle $2 \rho$, the sense of the rotation being counter-clockwise about the fast state $R$.

Figure 6 shows the evolution of the pendulum orbit from a rectilinear starting state $\mathrm{P}_{0}$, when both linear and circular biresonance are present. The phase rates of change $\dot{\delta}$ and $2 \dot{\rho}$ measure the relative amount of each type of biresonance vectorially, the vectors pointing toward their respective fast states. The vector sum of linear and circular biresonance defines the fast elliptic state $M$ and the rotation axis $M N$ for elliptic biresonance. The diametrically opposed states $M$ and $N$ are the elliptic orthogonal eigenstates with major axes at $90^{\circ}$ from each other and with ellipticities of opposite signs.

\subsection{Nonlinear Airy precession rate}

The Airy effect is due to the anharmonicity of the restoring force, since actual pendula are not true harmonic oscillators. It is a form of induced circular biresonance which is proportional to the area of an elliptical orbit. It has the same sign as the ellipticity, so that the precession direction is the same as the rotation direction of the pendulum bob along the orbit. By itself, the Airy effect does not change the ellipticity. Hence, a perfectly isotropic pendulum started with an elliptical initial state would have the ellipse precess without changing its shape, and the representative point $\mathrm{P}$ on the Poincare sphere would describe a small circle at constant latitude and constant velocity. The Airy precession rate is given by [9, 13]

$$
\Omega_{A}=3 \omega a b / 8 l^{2}
$$




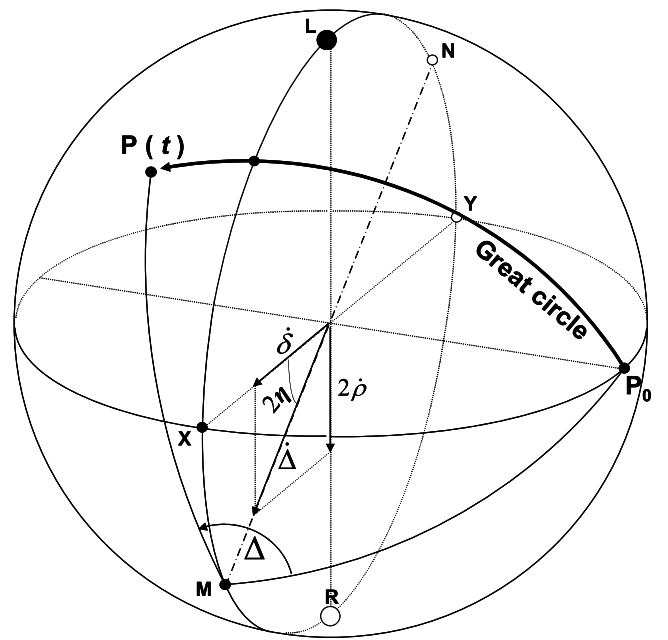

Fig. 6. Evolution of the pendulum orbit represented by point $\mathrm{P}$, with a rectilinear starting state $\mathrm{P}_{0}$, when both linear and circular biresonance are present. The phase rates of change $\dot{\delta}$ and $2 \dot{\rho}$ measure the relative amount of each one. Linear and circular biresonance add up quadratically to yield the amount of elliptic biresonance $\dot{\Delta}$.

where

$$
\begin{aligned}
\omega & =\text { pendulum swinging frequency; } \\
a, b & =\text { ellipse semi-axes; } \\
l & =\text { pendulum length. }
\end{aligned}
$$

Figure 7 illustrates the locus of the representative point $\mathrm{P}$ under the influence of Airy precession. The starting state 0 is a rectilinear vibration at an azimuth close to the slow axis $Y$. Since the pendulum already has a constant linear biresonance $\dot{\delta}$, rotation of point P clockwise (cw) about the slow eigenstate $Y$ brings it immediately into the northern hemisphere, which means the onset of a ccw elliptical orbit. The accompanying Airy precession rate at timestamp 1 changes the rotation axis from $X Y$ to $M_{1} N_{1}$. At the same time, the rotation rate (rate of phase change) increases since the module of $\dot{\Delta}$ increases, while on the locus of $\mathrm{P}$, the representative point also accelerates due to the longer radius about the instantaneous rotation axis $M_{1} N_{1}$. The rapidly moving rotation axis $M N$ away from the locus of $\mathrm{P}$ causes the ellipticity to saturate (here at timestamp 3.5) when P crosses the meridian of the characteristic biresonance eigenplane $X L Y R$. Airy precession, which is measured as twice the azimuth of point $\mathrm{P}$, always accelerates (or decelerates) toward the eigenplane and the slow state $Y$.

It is interesting to note that, for a harmonic oscillator (no Airy effect) with linear biresonance but in absence of Foucault effect (at the earth equator), the locus of $\mathrm{P}$ would be a small circle described at constant angular velocity on the Poincaré sphere. The precession angle would oscillate about the eigenstate $Y$ with an approximate sinusoidal speed between two extreme azimuths: the rectilinear state 0 and its symmetrical rectilinear state on the opposite side of $Y$. However, to the first order of this theory of the anharmonic oscillator, the locus of $\mathrm{P}$ is no longer a circle but an oval with its long axis along the equator of the Poincaré sphere. On that oval, $\mathrm{P}$ accelerates from state 0 , rushes through the eigenplane in the vicinity of state $Y$, decelerates to an about turn at the state symmetrical of 0 and then completes the cycle in the southern hemisphere with ellipses described in the opposite sense. 

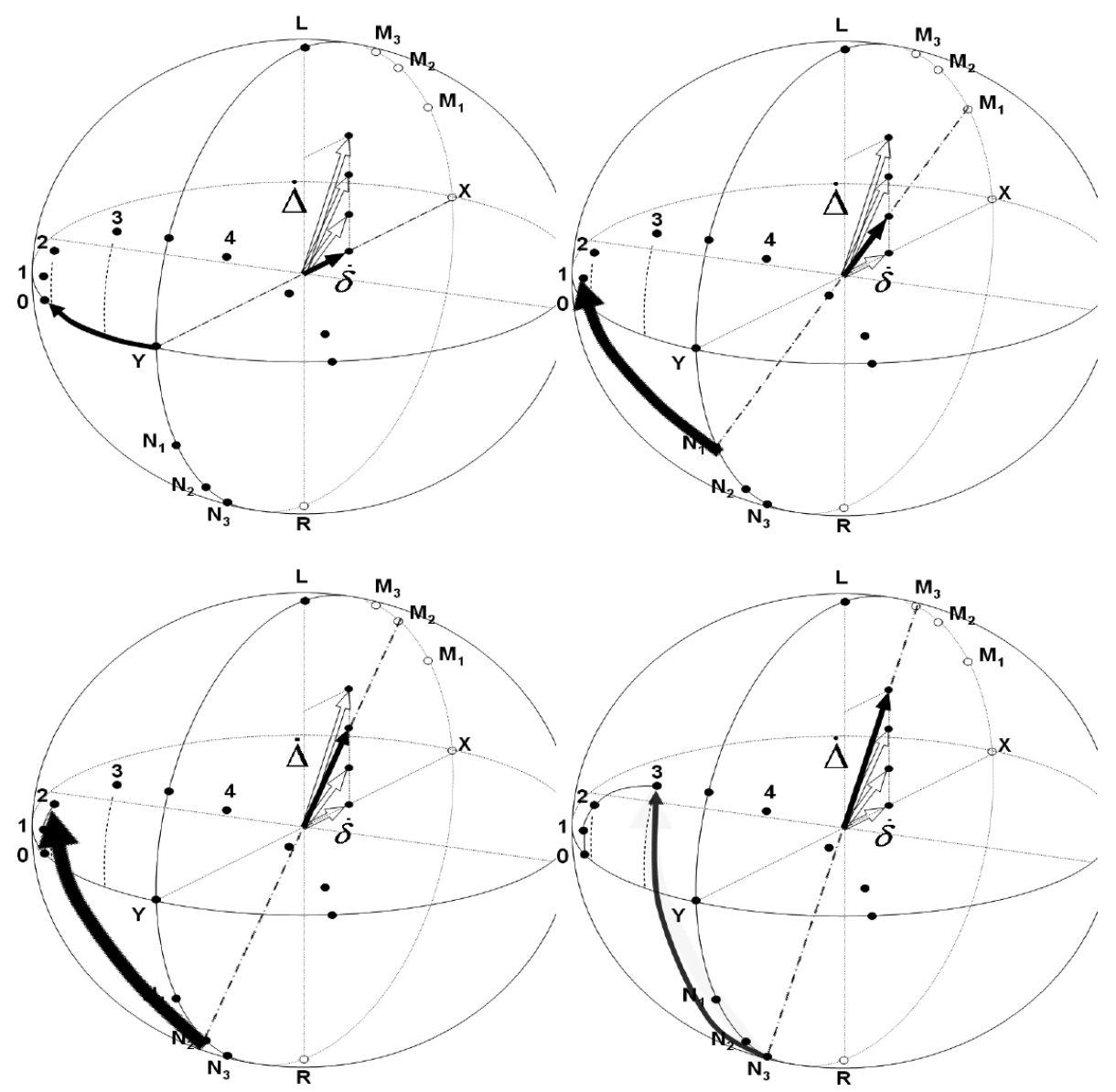

Fig. 7. Evolution of the pendulum orbit as represented by the sequence $0,1,2,3,4 \ldots$, with a rectilinear starting state 0 close to the slow eigenstate $Y$, when constant linear biresonance and induced Airy circular biresonance are present. The composite figure runs through 3 time increments. As soon as ellipticity sets in (time 1), Airy circular biresonance appears as a vertical (polar) component of phase rate of change, leading to a change in the rotation axis from $X Y$ to $M_{1} N_{1}$, and so on. The meridional points $Y, N_{1}, N_{2}, N_{3}$ show the sequences of the moving instantaneous centers of rotation of the radius vector describing the phase angle. The phase angle rate of change increases up to a maximum at time 3.5 , as the module of the ellipse phase rate vector $\dot{\Delta}$ (blackened) becomes maximal. The Airy contribution of circular biresonance is the polar component of $\dot{\Delta}$, which equals $2 \Omega_{A}$.

\subsection{Mixture of linear biresonance, Airy effect and Foucault effect}

The situation of Fig. 8 represents a good quality anisotropic paraconical pendulum in the course of a 14 minute run. The polar vector accounts for a typical mid-northern latitudes Foucault precession rate $\dot{\rho}=5 \cdot 10^{-5} \mathrm{rad} / \mathrm{s}$. The residual linear biresonance $\dot{\delta}$ amounts to $\sim 30 \%$ of the "cosmological" circular contribution $2 \dot{\rho}$. The slow elliptical eigenstate $N_{0}$ lies then way up near the L pole. $N_{0}$ will act as the first instantaneous rotation center for the radius vector joining the initial rectilinear state 0 and thereafter rotating cw about the $N$ 's. At the start, the locus of $\mathrm{P}$ will progress perpendicularly to the radius vector $N_{0}-0$ toward point 2 at essentially twice the Foucault precession rate, while $N_{0}$ slowly switches to $N_{2}$ during the first 2 minutes. The mobile state $N$ accelerates downward along the meridian of the eigenplane. After 7 minutes, the Airy precession rate completely compensates the Foucault rate, so that 


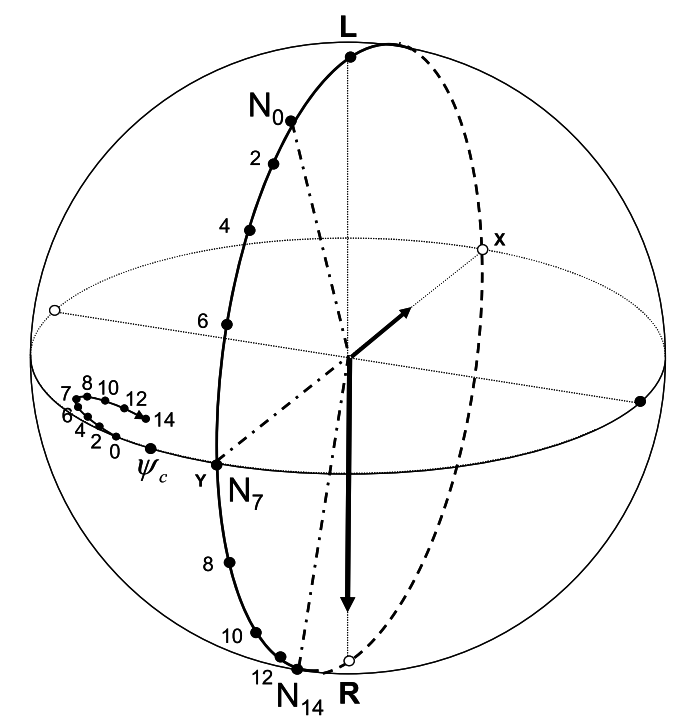

Fig. 8. Locus of point $P$ represented by the sequence $0,2,4, \ldots 12,14$, with a rectilinear starting state 0 close to the slow eigenstate $Y$, when constant linear biresonance, Foucault circular biresonance and induced Airy circular biresonance are present. A typical 14-minute run is considered. For the sake of clarity, the radius vectors linking the individual $N_{i}$ to their corresponding point $i$ on the locus of $\mathrm{P}$ are not shown. As soon as ellipticity sets in, Airy circular biresonance diminishes, nullifies and later overrides the Foucault circular biresonance vector shown. If the initial state 0 coincides with the critical azimuth $\psi_{c}$, then the final 14-minute azimuth comes back to the azimuth $\psi_{c}$.

the mobile rotation axis $M N$ coincides with the linear biresonance axis $X Y$. Point 7 marks the end of precession in the Foucault direction. The rapid passage of state $N$ through the equator of the Poincare sphere is responsible for the abrupt kink in the locus of $\mathrm{P}$ at point 7, after which the Airy precession rate supersedes the Foucault rate. The subsequent deceleration of $N$, as state $N_{14}$ is approached, causes a quasi-rectilinear segment of the locus of $\mathrm{P}$ at point 14 and ellipticity saturation is reached (see the lower curves of Fig. 9).

It can be seen in Fig. 8 that after 14 minutes, the overall precession angle is back to nearly zero. In fact, there exists a critical starting azimuth $\psi_{c}$ on the Foucault direction side of the eigenplane azimuth $Y$ where the 14-minute compensation is exact.

a) If the start state 0 lies within the interval $\left[\psi_{c}, Y\right]$, the initial segment of the locus is more parallel to the equator and the ellipticity grows less rapidly. The Airy precession also grows more slowly and it does not quite compensate the Foucault precession after 14 minutes. There remains a net precession angle toward $\psi_{c}$.

b) If the start state 0 outside the interval lies beyond state $\psi_{c}$, the initial segment of the locus makes a steeper angle with the equator, pointing into the northern hemisphere. The faster ccw ellipticity growth generates an overcompensating Airy precession which brings the final 14-minute azimuth closer to state $\psi_{c}$.

c) If the start state 0 outside the interval lies beyond state $Y$, the initial segment of the locus readily gains the southern hemisphere into the domain of the $\mathrm{cw}$ ellipses. Airy precession and Foucault precession both act in the same direction and tend to bring the pendulum azimuth toward the interval $\left[\psi_{c}, Y\right]$.

It is then clear that one can take advantage of that stabilizing property to experimentally determine the position of any unknown eigenplane and the direction of the linear biresonance vector $\dot{\delta}$. One only has 
to do chained experiments, where the start azimuth of run $(i+1)$ is the final azimuth of run $i$. No matter the first start azimuth, the sequence of 14-minute azimuths converges to the critical state $\psi_{c}$, where the offset $\left(\psi_{c}-Y\right)$ is essentially determined by the amount of local Foucault effect and by the geometry of the ellipsoid of inertia. At the earth equator, that procedure leads directly to the determination of the eigenstate $Y$.

\section{A new interpretation of Allais's pendulum experiments}

As mentioned in the introduction, Allais performed chained experiments with his paraconical short pendulum. The instrument involved with Figs 9 and 10, due to suspension anisotropy, has a characteristic linear biresonance in the range 10\%-20\% of the natural Foucault circular biresonance in Paris. The data of Fig. 9 originate from a 15-day calibration experiment with a spherical bob [28]. From the orientation of the suspension beams, the estimated low frequency state $Y$ should lie in the geographical azimuth of $206^{\circ}$, measured cw from north. All the chained experiments are started at an azimuth $45^{\circ}$ lower, namely $161^{\circ}$. Due to the Foucault effect and the Airy effect working in the same sense on that side of the low frequency state $Y$, it takes generally 5 to 7 runs for the 14-minute azimuths to cross over state $Y$ and oscillate about a mean azimuth of $212.3^{\circ}$, which can be regarded as the critical azimuth $\psi_{c}$. Beside the Foucault effect influence, the offset of $6.3^{\circ} \mathrm{cw}$ can also be visualized as connected to the depth of the saddle shaped gravitational potential surface created by the suspension geometry.

In Fig. 9, let us concentrate our attention on the precession (middle) curves. From typical chained experiments reported on p. 104 and p. 140 of Ref. [28], the standard error on each point, as the mean value from 540 individual measurements in columns (b) and (c), is of the order of $0,01 \mathrm{grad}$. Hence the values of $+0.07 \mathrm{grad}$ and $-0.15 \mathrm{grad}$ for the respective 14-minute azimuths in the morning hours and in the evening hours are highly significant. They are correlated with the relative position of the moon with respect with the position of the pendulum in that 15 day period.

Figure 10 shows a detailed variation of the 14-minute azimuth for a particularly significant experiment in June-July 1955. Allais's spectral analysis with a 24 solar hour cycle over 30 days yields an amplitude of 5.8 grad, while the adjustment over 28 lunar days gives 7.0 grad.

In general, all Allais's one-month experiments showed a lunar cycle with amplitudes of the order of $1^{\circ}$ in azimuth, except those of fall 1954 and summer 1955 (Fig. 10) with respective amplitudes of $5.8^{\circ}$ and $6.3^{\circ}$. In 1954, that was in the month preceding an eclipse. In the middle of the summer 1955 experiment, on June 20, there was an eclipse through Thailand and the Philippines where the sun-moon-earth syzygy was particularly good. Since it is now established from the above theory that the final 14-minute azimuth, except for a given offset, converges toward the low frequency eigenstate determined by environmental anisotropy of the pendulum, the variation of the 14-minute azimuth with a daily cycle suggests that the target eigenstate does undergo that daily cycle in azimuth with an amplitude of a few degrees.

The anisotropic pendulum behaviour can be interpreted in terms of shallow saddle potential superimposed over the spherical gravitational potential well. The low frequency eigenstate (longest swinging period) is in the azimuth of the line of local potential minima. An isolated mass in the pendulum environment does not show revolution symmetry about the pendulum axis. It will generate a saddle gravitational potential for the pendulum, where the line of local minima points toward that mass. In this way, the saddle potential due to the moon rotates under the pendulum according to the lunar day cycle. In order to be able to perturb the suspension anisotropy saddle by a few degrees daily, the lunar saddle must have a depth comparable with that of the suspension anisotropy saddle. However, independent calculations of the saddle potential from the moon by Allais [31] and by the present author fall short by $\sim 7-8$ 


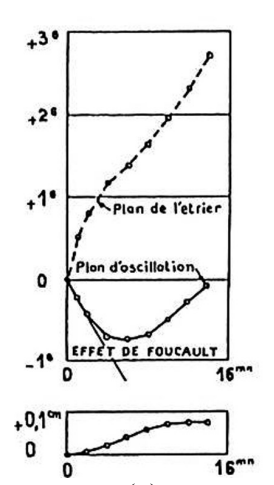

(a)

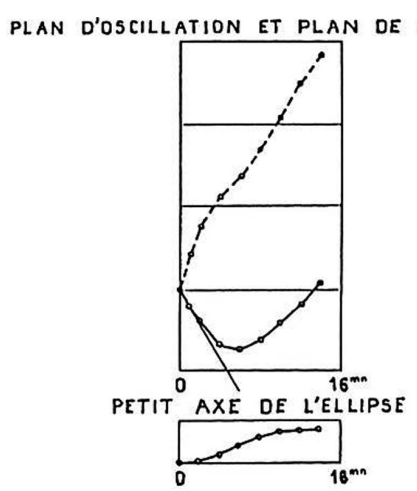

(b)

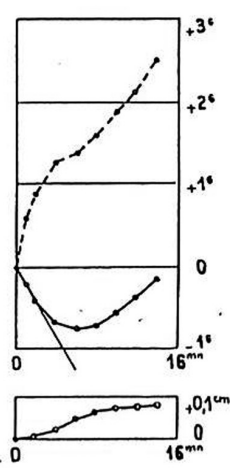

(c)

Fig. 9. Full day (a) and half day (b and c) averages of spin angle (upper curves), precession angle (middle curves) and minor axis values (lower curves) after 14 minutes for Allais's 15-day experiment Series VII, Sept. 21-Oct. 5, 1955. The angle units are grads. Columns (b) and (c) show the averages for the interval $0-12 \mathrm{~h}$ and the interval $12-24 \mathrm{~h}$ respectively. The Foucault rate is $\dot{\rho}=-2.94 \mathrm{grad} /(14 \mathrm{~min})=-2.64^{\circ} /(14 \mathrm{~min})=-5,5 \cdot 10^{-5} \mathrm{rad} / \mathrm{s}$ [29]. Note that the precession angle change becomes positive after 14 minutes during the morning hours (b), while it stays negative in the evening hours (c). Adapted from Allais' memoir to the NASA [30].

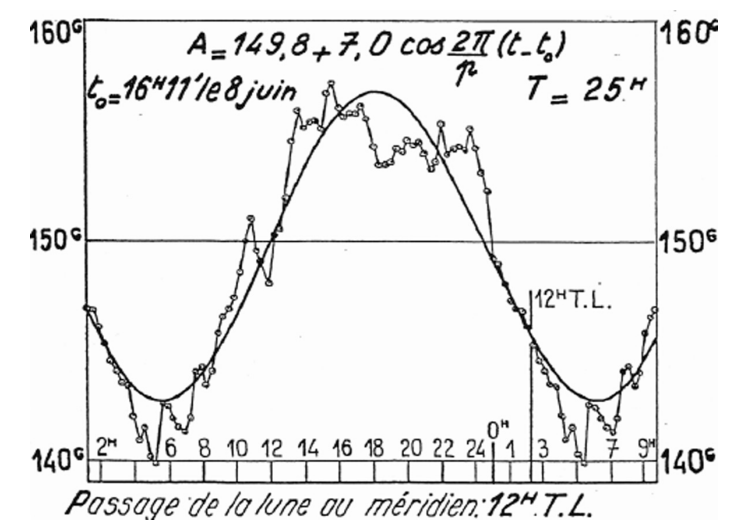

Fig. 10. Averaged 14-minute run azimuths as a function of the approximate lunar time ( 25 solar hours per lunar day instead of $24 \mathrm{~h} 50 \mathrm{~min}$ ) for the 30 day experiment from June 7 to July 7, 1955. The angle units are grads. The abscissa is labeled in mean solar time. Each point is an average over 28 lunar days. The 7.0 grad $\left(6.3^{\circ}\right)$ amplitude is very significant. (Graph reproduced from Ref. [28], p. 100).

orders of magnitude of the depth values for the suspension anisotropy saddle. Hence, the moon saddle potential cannot be held responsible for the observed oscillations of the anisotropy eigenstates $Y$. Those observations call for some unknown property of gravitation.

\section{Conclusion}

In fact, pendulum motion is more complex than what can be explained by summing many two-body problems: pendulum-earth, pendulum-moon, etc. In particular, the pendulum simultaneously lies in the far field of some distant masses and in the near field of an important mass distribution. As such, it should qualify for being addressed as an interior problem. Taking example with solid state physics and particle dynamics in solids, the concept of tensor effective mass has been successfully applied within 
Lorentz-Poincaré symmetry in crystals. It looks as if a tensor gravitational mass is needed, capable of describing accelerations not only along the classical line of action but also related to sideways motion. In kinematics, the Coriolis acceleration is successfully applied to the pendulum motion normal to a rotation axis. The equivalent link in dynamics appears to be missing. Santilli gravitation [32] is probably a good candidate to fill that gap.

\section{Acknowledgments}

The author has greatly appreciated beneficial discussions with Professor R.M. Santilli and particularly with Professor C. Corda. He is also grateful to the Santilli Foundation for partial financial support of this work.

\section{References}

[1] L. Foucault, Démonstration physique du mouvement de rotation de la terre au moyen du pendule, Comptes Rendus Hebdomadaires des Séances de l'Académie des Sciences 32 (3 Feb 1851), 135-138.

[2] G.B. Airy, On the vibration of a free pendulum in an oval differing little from a straight line, Proc Royal Astron Soc XX (9 May 1851), 121-130.

[3] H. Kamerlingh Onnes, Nieuwe bewijzen voor de aswenteling der aarde, Thesis, Rijksuniversiteit te Groningen, Groningen, $N L(1879), 1-290$

[4] A.S. Chessin, On Foucault's pendulum, Am J Math 17 (1895), 81-88.

[5] W.D. MacMillan, On Foucault's pendulum, Am J Math 37 (1915), 95-106.

[6] A.C. Longden, On the irregularities of motion of the Foucault pendulum, Phys Rev XIII (1919), 251-258.

[7] W.J. Noble, A direct treatment of the Foucault pendulum, Am J Phys 20 (1952), 334-336.

[8] W.B. Somerville, The description of Foucault's pendulum, Q J Royal Astron Soc 13 (1972), 40-62.

[9] M.G. Olsson, The precessing spherical pendulum, Am J Phys 46 (1978), 1118-1119.

[10] R. Latham, An interim report on a repeat of the Allais experiment - the measurement of the rate of increase of the minor axis of a Foucault pendulum using automatic apparatus, I C Report G28, Imperial College London January (1980), 70, Available PDF at: http://home.t01.itscom.net/allais/blackprior/latham/latham-rep28-1.pdf. Consulted May 18, 2010.

[11] K.T. Hecht, The Crane Foucault pendulum: An exercise in action-angle variable perturbation theory, Am J Phys 51 (1983), 110-114.

[12] R.A. Nelson and M.G. Olsson, The pendulum - rich physics from a simple system, Am J Phys 54 (1986), 112-121.

[13] A.B. Pippard, The parametrically maintained Foucault pendulum and its perturbations, Proc R Soc Lond $\mathbf{A 4 2 0}$ (1988), $81-91$.

[14] For a Foucault pendulum in an airbus, see: http://www.youtube.com/watch?v=ToUCXRnlfog\&feature=related. Consulted May 20, 2010.

[15] C.F. Moppert and W.J. Bonwick, The new Foucault pendulum at Monash University, Q J R Astron Soc 21 (1980), 108118.

[16] F. Charron, Bulletin de la SAF (Novembre 1931).

[17] M. Allais, Observation des mouvements du pendule paraconique, CRAS Tome 245 (1957), 1697-1700.

[18] M. Allais, Mouvement du pendule paraconique et élipse totale de soleil du 30 juin 1954, CRAS Tome 245 (1957), 20012003.

[19] C.P. Duif, A review of conventional explanations of anomalous observations during solar eclipses, arXiv:gr-qc/0408023 5 (31 Dec. 2004).

[20] H.A. Munera, Should the laws of gravitation be reconsidered? The scientific legacy of Maurice Allais, Apeiron Montreal Canada (2011), 447.

[21] E.J. Saxl and M. Allen, 1970 Solar Eclipse as "Seen" by a Torsion Pendulum, Phys Rev D 3 (1971), 823.

[22] T. Kuusela, Effect of the solar eclipse on the period of a torsion pendulum, Phys Rev D 43 (1991), 2041.

[23] M. Duval, An experimental gravimetric result for the revival of corpuscular theory, Phys Essays 18 (2005), 53-62. Results first presented at 69e Congrès de l'ACFAS, Sherbrooke, Qc., Canada, (13-17 May 1996).

[24] M. Duval, Vérification expérimentale de la théorie corpusculaire de la gravitation. http://arxiv.org/ftp/arxiv/papers/ 0705/0705.2581.pdf, (2007).

[25] H. Poincaré, Théorie mathématique de la lumière, Gauthier-Villars, Paris II(12) (1892). 
[26] R. Verreault, A new method to measure general birefringence in crystals, Zeitschrift für Kristallographie 136 (1972), 350-386.

[27] R. Verreault, Can Santilli gravitational anisotropy be detected via pendulum experiments? San Marino workshop on astrophysics and cosmology for matter and antimatter. San Marino, Italy, September 5-9, 2011. Lecture 10 on http:// www.world-lecture-series.org/san-marino-2011.

[28] M. Allais, L'Anisotropie de l’Espace, Clément Juglar, Paris (1997), 81-101.

[29] M. Allais, L'Anisotropie de l’Espace, Clément Juglar, Paris (1997), 93.

[30] M. Allais, The "Allais Effect" and my Experiments with the Paraconical Pendulum 1954-1960, a memoir prepared for NASA, 1999, available online at: http://www.allais.info/alltrans/nasarep.htm.

[31] M. Allais, L'Anisotropie de l'Espace, Clément Juglar, Paris (1997), 118-129.

[32] R.M. Santilli, Isominkowskian geometry for the gravitational treatment of matter and its isodual for antimatter, International Journal of Modern Physics D 7(3) (1998), 351-407. 